\title{
Automated Photosensitivity Enhancement in Optical
}

\section{Fiber Tapers}

\author{
Aleksander Sade Paterno* \\ Santa Catarina State University - Joinville Santa Catarina 89223-100 \\ *dee2asp@joinville.udesc.br \\ Valmir de Oliveira, Hypolito J. Kalinowski \\ Federal University of Technology - Paraná Curitiba, Paraná 80230-901 \\ valmir@utfpr.edu.br,hjkalin@cpgei.ct.utfpr.edu.br
}

\begin{abstract}
An alternative technique that uses a flame-brush at high temperature to enhance UV light photosensitivity in an optical fiber is described. An extreme low-cost air aspirated propane-butane mini-torch is used, which produces a lower temperature flame than the one in the flame-brush original technique. It is shown that this change in the previous technique is also capable of improving photosensitivity and allowing the fiber Bragg grating imprinting process to be accelerated. Since the flame-brush photosensitivity enhancement process is designed to operate in an automated fiber taper rig, the process was evaluated in optical fiber tapers with different diameters. In this case, changes in photosensitivity are observed in the tapers in addition to the intrinsic photosensitivity occurring in the pristine fiber without being tapered.
\end{abstract}

Index Terms - Fiber Bragg gratings, optical fiber taper, photosensitivity, flame-brush technique.

\section{INTRODUCTION}

Fiber Bragg Grating (FBG) imprinting processes form already a mature science [1],[2] and there are several types of fibers to be used in the fabrication of such devices. A preferred choice for the fabrication of FBG would be a standard single-mode optical fiber compatible with many devices used in fiber optic communications. Any germanium-doped silicate optical fiber core demonstrate some photosensitivity to UV light at different degrees[3] and could be used for the FBG fabrication, for example, when using an excimer laser at $248 \mathrm{~nm}$ in the imprinting process. There are several well established methods to enhance photosensitivity to inscribe FBG and consequently require shorter exposure times to the UV light and cause the reliability and spectral characteristics of the fabricated devices to be differently affected. A class of such techniques is based on the diffusion of Hydrogen via different methods into the fiber, like the hot hydrogenation and high-pressure cold hydrogenation, or the exposure of the bare fiber to a Hydrogen-Oxygen flame for 20 minutes[4],[1]. In this last case, the process is simple, requiring a scanning torch along the fiber at a temperature of approximately $1700^{\circ} \mathrm{C}$. The flame-brushing in the photosensitivity enhancement process is similar to the one used in the fabrication of fused biconical optical fiber tapers with the flame-brush technique [5],[6]. For the fabrication of fiber tapers, the heat-source is not required to be a Hydrogen-Oxygen flame, and even a commercial air-aspirated propane-butane mini-torch may allow the manufacture of the tapers [7]. 
The observation of photosensitivity enhancement along the flame-brushed fiber section is noticed in this work at a much lower temperature (approximately $1000^{\circ} \mathrm{C}$ ) even when using a Butane-Oxygen flame.

The photosensitivity enhancement in germanosilicate fibers is associated with germanium-oxygen deficient centers (GODC) whose concentration in the fiber structure depends on competing reactions, namely, the creation of GODCs and their destruction that would produce other defects in the glass network [8]. A temperature increase would shift the balance of the reactions to generate and destroy GODCs [9], which are also responsible for the absorption bands in the UV, at wavelengths used to imprint FBG, for example, at $242 \mathrm{~nm}$. A higher absorption in the UV band would imply a change in the refractive index values at longer wavelengths, according to the Kramers-Kronig relations [1], causing the production of the refractive index modulation and therefore the fiber Bragg grating.

Such a temperature increase to implement the commercial mini-torch photosensitivity enhancement process may be used in the fiber taper rig. It would require only slight changes in the control of the machine such as a software to allow only the flame-brushing of the fiber or the manufacture of tapers. Considering that such an enhancement in photosensitivity when imprinting the FBG with an excimer laser was first observed by the authors in a fiber optic taper, tapers with different diameters were analyzed with respect to the imprinting efficiency. Its photosensitivity was compared to the intrinsic photosensitivity observed in pristine fibers.

\section{METHODOLOGY}

The alternative flame-brush technique for the photosensitivity enhancement of an optical fiber was implemented in a fiber taper rig system to manufacture fused bi-conical optical fiber tapers [7]. The system operates by slowly stretching the optical fiber while a flame-brush moves in a zigzag movement along a hot-zone section of the fiber. If the photosensitivity enhancement is the goal in the system, the taper rig can be programmed to move only the flame-brush, allowing the uniform heating of the fiber during a specific time interval. The time duration of the flame-brushing process in the original technique is usually set to 20 minutes with a Hydrogen-Oxygen flame [4]. The flame-brush is based on a commercial mini-torch that after being refueled is able to maintain a flame for 40 minutes at a temperature of approximately $1000^{\circ} \mathrm{C}$ with a propane-butane $(30 \% / 70 \%)$ mixture.

A standard single-mode optical fiber was used (Draktel G.652.C/D) into which tapers were manufactured and FBG imprinted. A set of fiber tapers was fabricated, having exponential profile up to the waist region, which had constant diameters of $70 \mu \mathrm{m}, 90 \mu \mathrm{m}$ and $110 \mu \mathrm{m}$. All taper waists were processed along a length of $20 \mathrm{~mm}$, equal to the taper length, with the flame at a speed of $4 \mathrm{~mm} / \mathrm{s}$. After being heated, these fiber sections were exposed to the beam of a pulsed $\mathrm{KrF}$ excimer laser (Xantos XS-500, Coherent) with center wavelength at $248 \mathrm{~nm}, 8 \mathrm{~mJ}$ pulse energy and $5 \mathrm{~ns}$ pulse width at a rate of up to $500 \mathrm{~Hz}$. The Excimer laser beam traversed a phase-mask (with pitch of 1066.5 $\mathrm{nm}$ ) positioned close to the fiber, before hitting the taper waist region and resulting in the modulation 
of the refractive index in the core of the fiber. In fig. 1, a diagram illustrating this FBG imprinting process is depicted. This pattern of UV light on the fiber is able to produce an FBG with Bragg wavelength at approximately $1540.5 \mathrm{~nm}$. Therefore, the optical spectrum of the light reflected by the FBG is centered at approximately this wavelength.

The flame-brushed region of the fiber was the section into which the FBG should be imprinted. The number of Excimer laser pulses was recorded during the process together with the value of the reflectivity spectrum of the FBG by an interrogating unit (Micron Optics, Optical Sensing Interrogator SM 125). The reflectivity peak in the data set was measured to indicate how the refractive index in the core of the fiber is changing in an indirect manner, characterizing the FBG imprinting process through reflectivity peak changes. For each diameter a set of 5 tapers were produced to evaluate the repeatability of the observed effect, producing a mean value of reflectivity read with the interrogation system. A value associated with the reflectivity in decibels $(\mathrm{dB})$ is produced by calculating the difference between the base line of the spectrum data and its dip (or peak) in the transmission (or reflection) spectrum. A value of $25 \mathrm{~dB}$ would correspond to a reflectivity of $99.68 \%$. In order to illustrate the correspondence between the refractive index change and reflectivity, R, in the FBG one may use [10]:

$$
\mathrm{R}=\tanh ^{2}\left(\mathrm{~L} \pi \nu \delta \mathrm{n}_{\mathrm{eff}} / \lambda\right)
$$

where $\mathrm{L}=3 \mathrm{~mm}$ is the length of the imprinted $\mathrm{FBG}, \delta \mathrm{n}_{\mathrm{eff}}$ is the mean effective index variation caused during the FBG fabrication, $\lambda$ is the wavelength and $v$ is the fringe visibility of the index change.

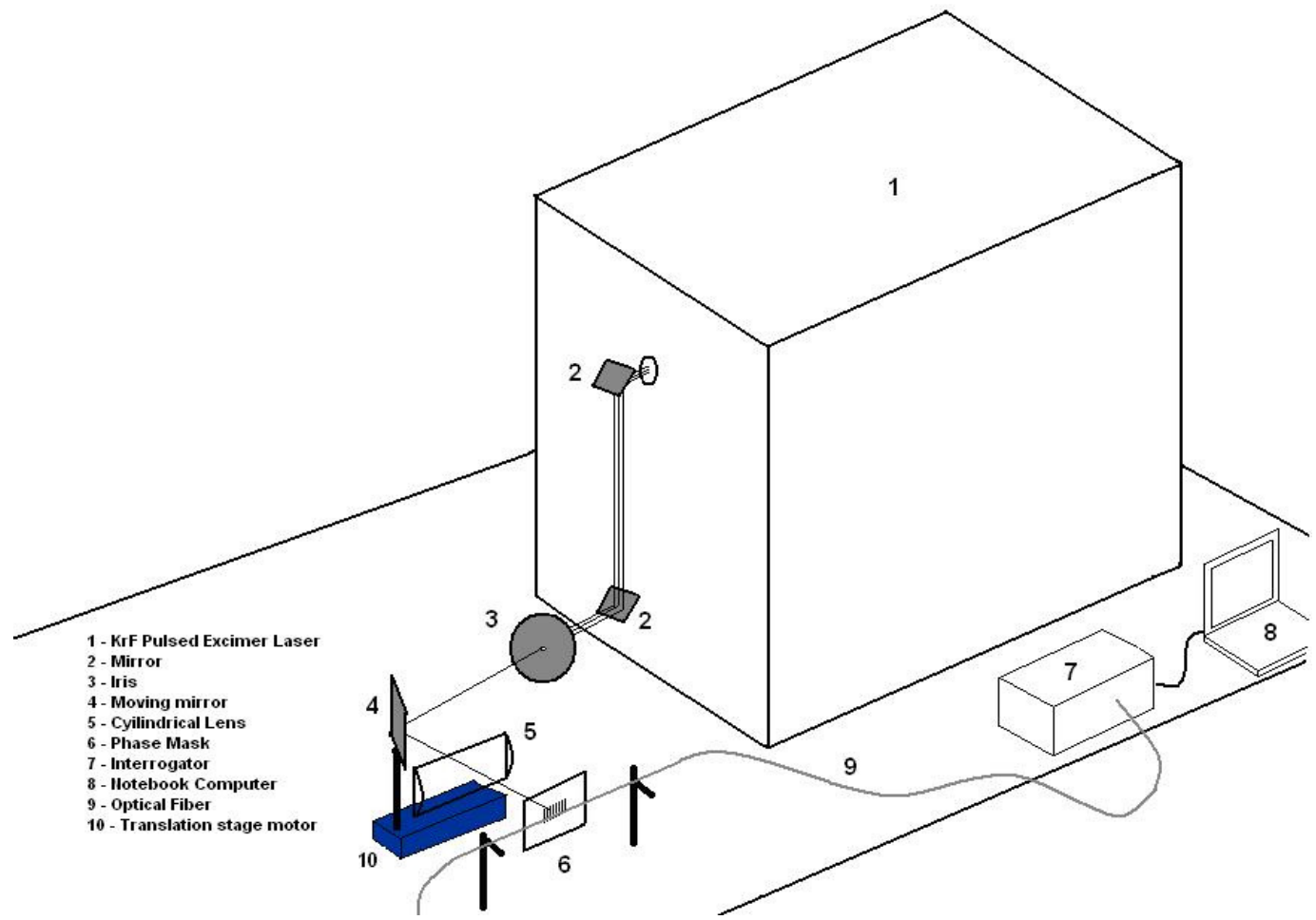

Fig. 1. Diagram illustrating the FBG fabrication: excimer laser, optical circuit and interrogating system. 
The $\delta n_{\text {eff }}$ could be calculated by fitting a simulated spectrum to the acquired reflectivity spectrum. The acquired spectrum data was processed using a radial basis function neural network to detect the correct position of the reflectivity peak and its wavelength [11].

In order to isolate the causes of the photosensitivity enhancement, another similar set of fibers were only flame-brushed for a period of time equal to the tapering process for the fabrication of tapers with $60 \mu \mathrm{m}, 85 \mu \mathrm{m}$ and $110 \mu \mathrm{m}$. The reflectivity of the FBG imprinting process was monitored after the transient of reflectivity growth, such as to show evidences of photosensitivity enhancement due to the tapering or to the flame-brushing.

\section{RESULTS}

During the FBG imprinting process in tapers, the peak reflectivity behavior was recorded as a function of the number of Excimer laser pulses, and this behavior is illustrated by the curves shown in fig. 2. An FBG was written in the pristine fiber that was not subjected to the flame-brushing process and its reflectivity behavior is used as a reference. At a number of $\mathrm{N}=1250$ pulses from the Excimer laser the peak reflectivity of each FBG was registered in normalized values and is depicted in table 1, showing the transient behavior of the reflectivity growth in absolute values. In order to illustrate the behavior of the FBG spectrum during the imprinting process two spectra obtained from an FBG imprinted in tapers with $110 \mu \mathrm{m}$ exposed to $\mathrm{N}=200$ and $\mathrm{N}=11200$ laser pulses and are depicted in fig. 3. The FBG reflectivity behavior during the process is monitored for flame-brushed and non-flamebrushed fibers with different taper diameters as a function of laser pulses number are also depicted in fig. 4 and 5.

Since the $8 \mathrm{~mJ}$ energy pulses are below the threshold of $30 \mathrm{~mJ}$, the written FBG were all in the type I regime of fabrication [1].

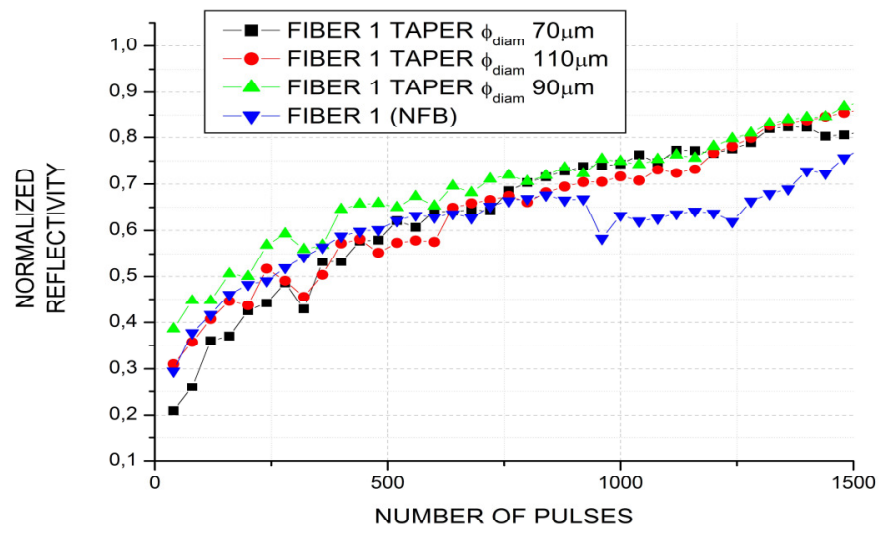

Fig. 2. Reflectivity of tapers and not-flamed brushed fiber (NFB) as a function of the number of excimer laser pulses during the FBG writing process. 


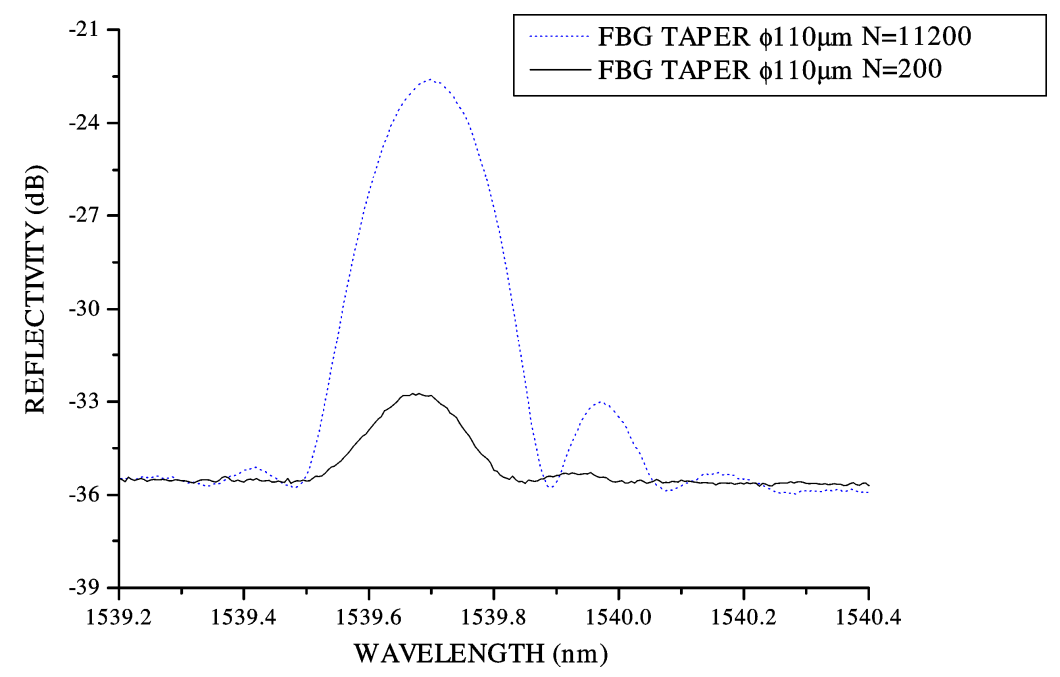

Fig. 3. Spectrum of the light reflected by the FBG in a taper with $110 \mu \mathrm{m}$ occurring at a number of pulses of N=200 (solid line) and $\mathrm{N}=11200$ (dotted line) from the Excimer laser.

TABLE I. TIME DURATION OF FLAME-BRUSHING PROCESS IN TAPERS AND NOT FLAME-BRUSHED (NFB) FIBERS AND THEIR CORRESPONDING REFLECTIVITY FOR 1250 EXCIMER LASER PULSES IN THE FBG WRITING.

\begin{tabular}{ccc}
\hline Diameter & Duration & Reflectivity at N=1250 pulses \\
\hline $125 \mu \mathrm{m}$ & Not flame-brushed & $61.9 \%$ \\
$110 \mu \mathrm{m}$ & $2.77 \mathrm{~min}$ & $79.7 \%$ \\
$90 \mu \mathrm{m}$ & $5.95 \mathrm{~min}$ & $78.1 \%$ \\
$70 \mu \mathrm{m}$ & $10 \mathrm{~min}$ & $77.6 \%$
\end{tabular}

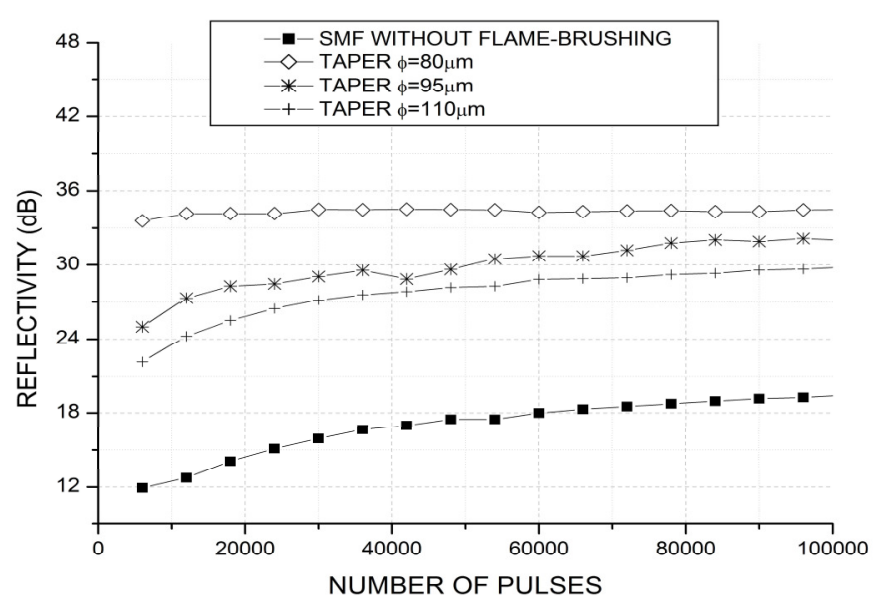

Fig. 4. Reflectivity behavior for FBG imprinted in tapered fibers with diameters of $80 \mu \mathrm{m}, 95 \mu \mathrm{m}$ and $110 \mu \mathrm{m}$ and in a standard single-mode fiber not subjected to the flame-brushing. 


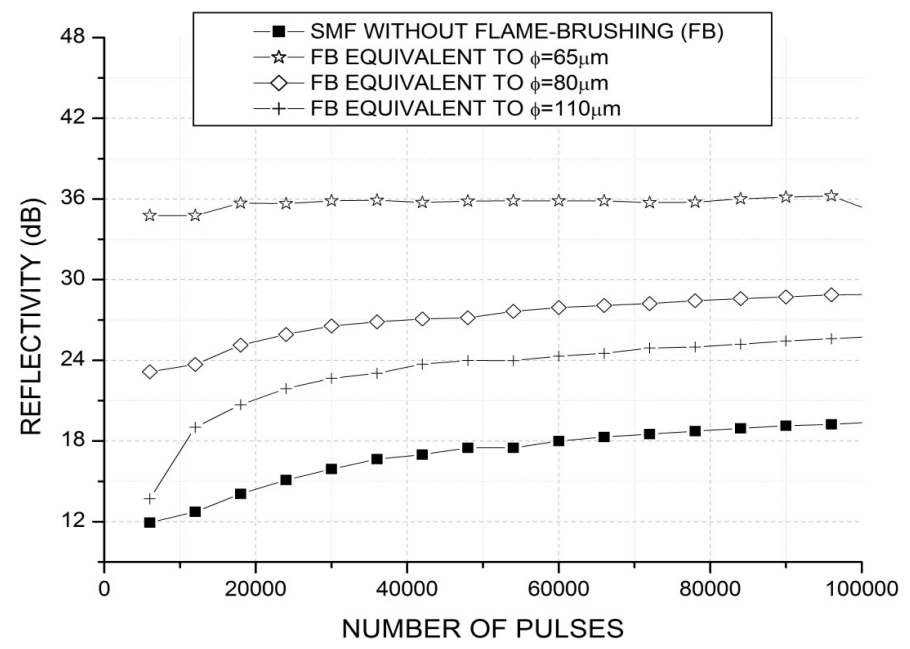

Fig. 5. Reflectivity behavior for FBG imprinted in flame-brushed fibers with a process duration equivalent to fabricating tapers with diameters of $65 \mu \mathrm{m}, 80 \mu \mathrm{m}$ and $110 \mu \mathrm{m}$, and in a standard single-mode fiber not flame-brushed.

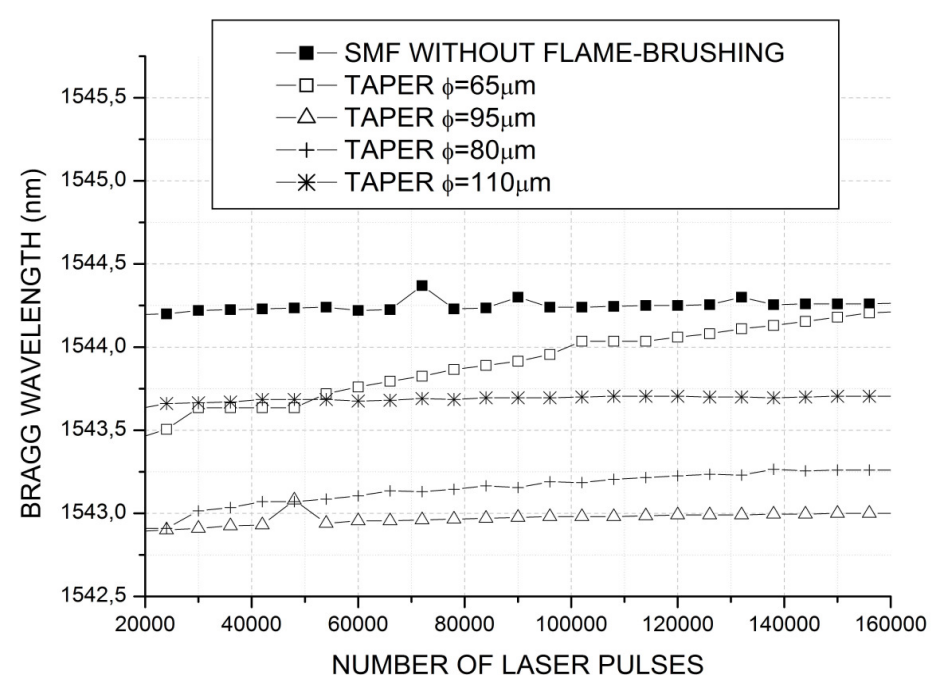

Fig. 6. Bragg wavelength behavior while imprinting FBG in tapers with diameters of $65 \mu \mathrm{m}, 80 \mu \mathrm{m}, 95 \mu \mathrm{m}$ and $110 \mu \mathrm{m}$, and in a standard single-mode fiber not subjected to flame-brushing.

A further study on the behavior of the FBG fabrication process in the taper for a larger number of laser pulses was implemented, where the dependence of the photosensitivity on the taper diameter was evaluated. In the case of the flame-brushing process, its duration in the manufacture of tapers with diameter $110 \mu \mathrm{m}, 90 \mu \mathrm{m}$ and $70 \mu \mathrm{m}$ is shown in table $\mathrm{I}$.

The Bragg wavelength of the FBG imprinted in tapers was also monitored as a function of number of laser pulses, to observe the relation between the taper diameter and the refractive index changes during the FBG inscription process. These curves are depicted in Fig. 6, for tapers with diameters of $110 \mu \mathrm{m}, 95 \mu \mathrm{m}, 80 \mu \mathrm{m}, 65 \mu \mathrm{m}$ and for a single-mode fiber that was not flame-brushed. 


\section{DISCUSSION}

From the results plotted in fig. 2, one may observe a relative improvement in photosensitivity that allows an accelerated FBG imprinting process in the fiber tapers. An illustration of the acquired FBG spectrum during the FBG writing is depicted in fig. 3 for the taper with $110 \mu \mathrm{m}$. The peak reflectivity behavior in any fiber taper shows a smooth increase in its mean value along the process, which remains above the pristine fiber reflectivity. The pristine fiber had an FBG imprinted using its intrinsic photosensitivity based on a low germanium core. It was also possible to notice that fiber tapers with smaller diameters tend to demonstrate a sudden decrease in the reflectivity, earlier than in tapers with larger diameters. This may be caused by the fiber annealing for a duration of the flamebrush longer than in the fabrication of tapers with larger diameters. The literature reports such an earlier erasure of the FBG written in annealed fibers and setting a threshold between the type I and type IIA FBG writing regime [12]. However, when carefully analyzing the acquired the data, since tapers are more sensitive to environmental disturbance, any perturbation in the taper position in the fiber holder during the FBG imprinting may cause the FBG to blur and consequently this may erase the refractive index modulation, increasing its mean level. One evidence of this blurring is depicted by the Bragg grating wavelength increase during the FBG imprinting in fig. 6. It was observed that the sensitivity of the imprinting process in fiber tapers to mechanical disturbances in the fiber may cause the FBG to blur and have its reflectivity suddenly decreased. It is natural that such a sensitivity of the FBG fabrication increases when smaller fiber taper diameters are used, since it is more difficult to position smaller tapers appropriately in the fiber holder.

A tendency to decrease the peak reflectivity is also partly noticed in the taper with $90 \mu \mathrm{m}$ which starts to decrease with $\mathrm{N}=1750$, supposedly due to such a disturbance. A flame-brushed fiber taper during 2.77 minutes shows also an increase in the reflectivity, and it follows the other FBG tapers reflectivity until $\mathrm{N}=1250$, that is determined in this fiber set as a threshold for comparison with tapers subjected to a longer flame-brushing process duration. This also indicates that the propane-butane flame allows the improvement of photosensitivity, while the process may be implemented with a simpler flame-brushing system in an automated taper rig.

In fig. 4, the experimental curves representing the mean value reflectivity for tapered fibers and for a reference pristine fiber that was not subjected to the flame-brushing process, as a function of the number of laser pulses, show the expected increase in the reflectivity during the FBG imprinting. Maximum reflectivity in the sample set is observed for the $80 \mu \mathrm{m}$ diameter that saturates its value at approximately $35 \mathrm{~dB}$. In fig. 5, similar experimental curves show the photosensitivity improvement associated with the flame-brushing process only, for a time duration equivalent to the fabrication of the tapers having the three largest diameters in the evaluated set. By observing fig. 5 one may assert that the photosensitivity improvement is not as pronounced as in fiber tapers. In order to obtain a saturated reflectivity as large as in the $80 \mu \mathrm{m}$ diameter taper, the pristine fiber must be flame-brushed as it would be when the $65 \mu \mathrm{m}$ diameter taper is manufactured. A first consideration would be that the 
tapering may cause the fiber to be slightly more photosensitive than the ordinary flame-brushed fibers. However other issues may be involved in this photosensitivity enhancement process, namely, the fact that smaller taper diameters may cause the coupling coefficient in the FBG [1] to change such as to cause a reflectivity enhancement, explaining the superior increase in reflectivity in fiber tapers. Therefore, it is possible that this enhanced photosensitivity in the tapers would be associated with the geometrical changes in the fiber core diameter and also the already known GODC defects generation occurring in the germanium-doped silica core during the flame-brushing.

In fig. 6, the Bragg wavelength of FBG in tapers with different diameters show that the mean refractive index variations during the imprinting process are more pronounced for smaller taper diameters. In tapers with a 125 and $110 \mu \mathrm{m}$ diameter, such changes are not observed, but they increase as the taper diameter decreases, partly explaining the blurring of the FBG, since an increased mean modulation index can be associated with the blurring of the FBG modulation index profile.

\section{CONCLUSIONS}

The automated flame-brushing of fiber optic tapers was demonstrated in a modified operation of a fiber taper rig. In addition, the used flame-brush is based on a commercial air aspirated propanebutane mini-torch, showing that there will be an increase in photosensitivity if the Hydrogen-Oxygen flame is not the used flame-brush. Other results about the increased photosensitivity in fiber optic tapers are reported, as the mechanical disturbances in the FBG imprinting and its behavior with different tapers, or equivalently with different time interval exposures to the flame-brushing process.

A further study is required in order to evaluate the FBG writing process in other flame-brushed fibers different from the standard single-mode fiber used in this work, as well as a more complete characterization of the FBG imprinted in the tapers and a comparison between their behavior and the FBG in flame-brushed optical fibers.

A photosensitivity enhancement was observed in optical fiber tapers with different taper diameters. The flame-brushing process occurring during the taper manufacture is known to produce a photosensitivity enhancement to imprint fiber Bragg gratings, but in optical fiber tapers the geometrical changes in the taper diameter also change the characteristics of the FBG inscription. Differently from the fabrication in ordinary single-mode fibers or in tapers with diameters of values close to the single-mode fiber diameters, the characteristics of the fabricated FBG as well as the efficiency of its fabrication are markedly altered, as discussed in this work.

\section{ACKNOWLEDGMENT}

The authors gratefully acknowledge the support of The National Council of Technological and Scientific Development (CNPq), and the support with the fiber taper rig software and electronic circuitry of the students Alexandre Felipe and Jean Graf. The Photorefractive Devices Unit (NUFORE) - UTFPR acknowledges financial support received from CNPq, FINEP and Fundação Araucária. 


\section{REFERENCES}

[1] R. Kashyap, Fiber Bragg Gratings. San Diego, EUA: Academic Press, 1999.

[2] A. Othonos and K. Kalli, Fiber Bragg Gratings: Fundamentals and Applications in Telecommunications and Sensing. Boston: Artech House, 1999.

[3] J. Stone, "Photorefractivity in GeO2-doped silica fibres," J. Appl. Phys., vol. 62, no. 11, p. 4371, 1987.

[4] F. Bilodeau, B. Malo, J. Albert, D. C. Johnson, K. O. Hill, Y. Hibino, M. Abe, and M. Kawachi, "Photosensitization of optical fiber and silica-onsilicon/silica waveguides," Opt. Lett., vol. 18, no. 12, pp. 953-955, 1993.

[5] R. P. Kenny, T. A. Birks, and K. P. Oakley, "Control of optical fibre taper shape," Electronics Letters, vol. 27, pp. 1654-1656, 1991.

[6] T. A. Birks and Y. W. Li, "The shape of fiber tapers," J. Lightwave Technol., vol. 10, no. 4, pp. 432-438, 1992.

[7] J. Graf, P. V. de Barba, J. Dallmann, J. A. S. Lima, S. A. Teston, H. J. Kalinowski, and A. S. Paterno, "Fiber taper rig using a simplified heat source and the flame-brush technique," in SBMO/IEEE MTT-S International Microwave and Optoelectronics Conference (IMOC), vol. 5622, 2009, pp. 621 - 624.

[8] G. Brambilla, V. Pruneri, L. Reekie, and D. N. Payne, "Enhanced photosensitivity in germanosilicate fibers exposed to $\mathrm{CO}_{2}$ laser radiation" Opt. Lett., vol. 24, no. 15, pp. 1023-1025, 1999.

[9] G. Brambilla and V. Pruneri, "Enhanced photosensitivity in silicate optical fibers by thermal treatment" Appl. Phys. Lett., vol. 90, issue 11, pp. 11905-1-3, 2007.

[10] T. Erdogan, "Fiber grating spectra," J. Lightwave Technology, vol. 15, no. 8, pp. 1227-1294, 1997.

[11] A. S. Paterno, J. C. C. Silva, M. S. Milczeswki, L. V. R. Arruda, and H. J. Kalinowski, "Radial-basis function network for the approximation of FBG sensor spectra with distorted peaks," Meas. Sci. Technol., no. 17, p. 1039-1045, 2006.

[12] M. Douay, W. X. Xie, T. Taunay, P. Bernage, P. Niay, P. Cordier, B. Poumellec, L. D. J. R. Bayon, H. Poignant, and E. Delevaque, "Densification involved in the UV based photosensitivity of silica glasses," J. Lightwave Technol., vol. 15, no. 8, pp. 1329-1342, 1997. 